\section{Día Mundial del Riñón}

\section{World Kidney Day}

El 13 de marzo de 2014 se celebra la 9a versión del World Kidney Day, evento anual patrocinado por la International Society of Nephrology y la International Federation of Kidney Foundations. El tópico en esta oportunidad es: "Chronic kidney disease and the ageing population".

Los párrafos siguientes se extrajeron de un documento ad hoc elaborado por los Drs. Marcello Tonelli (University of Alberta, Alberta, Canada) y Miguel Riella (Catholic University of Parana, Curitiba, Brazil):

"Chronic kidney disease (CKD) is an important public health problem that is characterized by poor health outcomes and very high health care costs. Since the prevalence of CKD is higher in older people, the health impact of population ageing will depend in part on how the kidney community responds."

"Socioeconomic development and increasing prosperity lead to ... increases in life expectancy. Older people account for a larger proportion of the general population".

"These demographic changes may have dramatic implications for conditions such as CKD, for which the prevalence increases with age. Estimated glomerular filtration rate (eGFR) declines with increasing age, and the incidence of dialysisdependent kidney failure has steadily increased among older people over the last few decades".

"Older people with CKD are at increased risk of adverse outcomes but they can still benefit from timely specialist referral; potential benefits include slower loss of kidney function (potentially preventing kidney failure), lower risk of cardiovascular events, and better information about renal replacement therapy".

"Although a large proportion of octogenarians who initiate dialysis will die within 6 months, a substantial minority may live for years. This heterogeneity in mortality is driven by differences in baseline co morbidity; when functional status is lower at baseline, initiation of dialysis often signals the onset of further decline. Nonetheless, quality of life appears reasonable among selected older dialysis patients - and can remain stable despite substantial co morbidity. Dialysis is therefore an appropriate treatment option for well-informed older people with kidney failure - especially for those with good baseline quality of life. Nevertheless good clinical judgment and careful communication will be increasingly required to support older people with CKD".

"Older age alone does not preclude kidney transplantation, which appears to reduce mortality among selected recipients, regardless of age. Use of expanded criteria deceased donors as well as more liberal use of older living donors also reduce mortality among older people with kidney failure, as compared to similar patients who remain on the transplant waiting list. The development of transplantation has great potential for developing countries, where dialysis may be unavailable or unaffordable".

"In summary, older people now account for a much greater proportion of patients with or at risk for kidney disease and kidney failure. The tremendous heterogeneity within this population requires discerning clinical management; chronological age alone is not a sufficient basis for clinical decisions, and a more nuanced approach is required - based on the comorbidities, functional status, quality of life, and preferences of individual patients. Dialysis and kidney transplantation can increase life expectancy - and will allow reasonable quality of life in selected older people with kidney failure. Perhaps more importantly, clinicians, patients and their families can be comforted by the knowledge that timely specialist evaluation can help to improve outcomes and reduce symptoms in older people with advanced kidney disease". 